\title{
A NOTE ON BEUKERS' INTEGRAL
}

\section{MASAYOSHI HATA}

(Received 18 May 1992)

Communicated by J. H. Loxton

\begin{abstract}
The aim of this note is to give a sharp lower bound for rational approximations to $\zeta(2)=\pi^{2} / 6$ by using a specific Beukers' integral. Indeed, we will show that $\pi^{2}$ has an irrationality measure less than 6.3489 , which improves the earlier result 7.325 announced by D. V. Chudnovsky and G. V. Chudnovsky.
\end{abstract}

1991 Mathematics subject classification (Amer. Math. Soc.): $11 \mathrm{~J} 82$.

\section{Introduction}

After Apéry's remarkable irrationality proofs for $\zeta(2)$ and $\zeta(3)$, Beukers [1] gave elegant proofs using the Legendre polynomials $L_{n}(x)=\left(x^{n}(1-x)^{n}\right)^{(n)} / n !$. Our aim in this note is to give a sharp lower bound for rational approximations to $\zeta(2)=\pi^{2} / 6$ using Beukers' double integral

$$
\iint_{S} \frac{F(x) G(y)}{1-x y} d x d y,
$$

where $S$ is the unit square $[0,1] \times[0,1]$ and $F(x), G(y)$ are non-zero polynomials with integral coefficients. Let $D_{n}$ be the least common multiple of $\{1,2, \ldots, n\}$ and let $\operatorname{ord}_{0}(F)$ be the order of the zero point of $F(x)$ at the origin. (We put $\operatorname{ord}_{0}(F)=0$ if $F(0) \neq 0$.) The double integral $(1.1)$ is very important in the arithmetical study of $\zeta(2)$ by virtue of the following lemma due to Beukers:

LEMMA 1.1. We have

$$
\iint_{S} \frac{F(x) G(y)}{1-x y} d x d y=a \zeta(2)+b,
$$

(C) 1995 Australian Mathematical Society 0263-6115/95 \$A2.00+0.00 
where

$$
a=\frac{1}{2 \pi i} \int_{C} F(z) G\left(\frac{1}{z}\right) \frac{d z}{z}
$$

is an integer, $C$ denotes a closed curve enclosing the origin and $b$ is a rational number whose denominator is a divisor of $D_{N} D_{M}$ with $M=\max \{\operatorname{deg}(F), \operatorname{deg}(G)\}$ and

$$
N=\min \left\{\max \left\{\operatorname{deg}(F), \operatorname{deg}(G)-\operatorname{ord}_{0}(F)\right\}, \max \left\{\operatorname{deg}(G), \operatorname{deg}(F)-\operatorname{ord}_{0}(G)\right\}\right\} .
$$

Note that the expression for $N$ in the above lemma comes from [1, Lemma 1]. In fact, the rational part in the right-hand side of the formula

$$
\iint_{S} \frac{(x y)^{n}}{1-x y} d x d y=\zeta(2)-\frac{1}{1^{2}}-\cdots-\frac{1}{n^{2}}
$$

belongs to the set $\mathbb{Z} / D_{N}^{2}$ since $n \leq \min \{\operatorname{deg}(F), \operatorname{deg}(G)\} \leq N$. (Note that always $M \geq N$.) Similarly, in the formula

$$
\iint_{S} \frac{x^{n} y^{m}}{1-x y} d x d y=\frac{1}{|n-m|}\left(\frac{1}{\min \{n, m\}+1}+\cdots+\frac{1}{\max \{n, m\}}\right)
$$

for $n \neq m$, the right-hand side belongs to the set $\mathbb{Z} / D_{N} D_{M}$, since

$$
|n-m| \leq \max \left\{\operatorname{deg}(F)-\operatorname{ord}_{0}(G), \operatorname{deg}(G)-\operatorname{ord}_{0}(F)\right\} \leq N
$$

and $\max \{n, m\} \leq M$, as required.

Beukers has studied the integral (1.1) with $F(x)=L_{n}(x)$ and $G(y)=(1-y)^{n}$, which produces a good irrationality measure for $\pi^{2}$ :

$$
\mu=1+\frac{\log (2+\sqrt{5})+6 / 5}{\log (2+\sqrt{5})-6 / 5}=11.85078 \ldots
$$

Taking more complicated polynomials in (1.1), the above measure $\mu$ has been pared down to 10.02979 . . by Dvornicich and Viola [4], to 7.552 by Rukhadze [9], to 7.5252 by the author [6], to 7.398537 by Rhin and Viola [8] and to 7.325 by Chudnovsky and Chudnovsky [2]. (The measures 7.552 and 7.325 were announced without proofs.)

In this note we consider the following integral

$$
\epsilon_{n}=\iint_{S} \frac{(x(1-x))^{15 n}(y(1-y))^{14 n}}{(1-x y)^{12 n+1}} d x d y
$$

instead of (1.1), which will produce a better irrationality measure of $\pi^{2}$ in the following sense: 
THEOREM 1.2. There exists a positive integer $q_{0}$ such that

$$
\left|\pi^{2}-\frac{p}{q}\right| \gg q^{-6.3489}
$$

for all $p \in \mathbb{Z}$ and any integer $q \geq q_{0}$. (In other words, $\pi^{2}$ has an irrationality measure less than 6.3489.)

We thus have immediately

COROLLARY 1.3. There exists a positive integer $q_{1}$ such that

$$
\left|\pi-\frac{p}{q} \sqrt{k}\right| \gg q^{-12.6978}
$$

for all $p \in \mathbb{Z}$ and any integer $q \geq q_{1}$ uniformly in $k$. ( $\pi / \sqrt{k}$ has an irrationality measure less than 12.6978.)

This will be proved using the same techniques as in [6, Corollary 5.2]. However, better irrationality measures for $\pi / \sqrt{k}$ for some particular integral values of $k$ are known; for example, $8.016045 \ldots$ and $4.601579 \ldots$ for $k=1$ and $k=3$ respectively (the author [7]), and $12.11 \ldots$ for $k=640320$ (Chudnovsky and Chudnovsky [3]).

After $12 n$-fold partial integration with respect to $x$, we have, from (1.2),

$$
\epsilon_{n}=\iint_{S} \frac{U(x) V(y)}{1-x y} d x d y
$$

with $U(x)=\left(x^{15 n}(1-x)^{15 n}\right)^{(12 n)} /(12 n) !$ and $V(y)=y^{2 n}(1-y)^{14 n}$. Hence it follows from Lemma 1.1 that

$$
\epsilon_{n}=a_{n} \zeta(2)+b_{n}
$$

where $a_{n} \in \mathbb{Z}$ and $b_{n} \in \mathbb{Z} / D_{16 n} D_{18 n}$, since $\operatorname{ord}_{0}(U)=3 n$ and $\operatorname{ord}_{0}(V)=2 n$.

In Section 2 we will study the asymptotic behaviour of $\left|\epsilon_{n}\right|$ and $\left|a_{n}\right|$. The most essential part of this note is Sections 3 and 4 , in which we will show that the denominator of $b_{n}$ is comparatively small; more precisely, $b_{n} \in\left(d_{n} / D_{16 n} D_{18 n}\right) \mathbb{Z}$ for some large product $d_{n}$ consisting of prime numbers less than $15 n$. Then Theorem 1.2 will be proved in Section 5 .

\section{Estimates of $\epsilon_{n}$ and $a_{n}$}

From (1.2) it is easily verified that

$$
\lim _{n \rightarrow \infty} \frac{1}{n} \log \left|\epsilon_{n}\right|=\max _{0<x, y<1} \log \left(\frac{(x(1-x))^{15}(y(1-y))^{14}}{(1-x y)^{12}}\right) .
$$


Since the maximum $\alpha$ of the right-hand side is attained at $x=(\sqrt{6969}-27) / 96$ and $y=(3 \sqrt{6969}-127) / 208$, we get

$$
\begin{aligned}
\alpha & =\log \left(\frac{5^{15} 7^{14}(\sqrt{6969}+21)^{12}(5 \sqrt{6969}-343)^{15}(99 \sqrt{6969}-7519)^{14}}{2^{257} 3^{15} 13^{28} 17^{12}}\right) \\
& <-36.0223
\end{aligned}
$$

We now estimate $\left|a_{n}\right|$. Since

$$
\begin{aligned}
a_{n} & =\frac{1}{2 \pi i} \int_{C} U(z) V\left(\frac{1}{z}\right) \frac{d z}{z} \\
& =\left(\frac{1}{2 \pi i}\right)^{2} \int_{C} \int_{C_{z}} \frac{(w(1-w))^{15 n}}{(w-z)^{12 n+1}} d w \frac{(1-z)^{14 n}}{z^{16 n+1}} d z,
\end{aligned}
$$

where $C$ and $C_{z}$ are circles centered at $z=0$ and $w=z$ with radii $r$ and $\rho$ respectively, we have

$$
\limsup _{n \rightarrow \infty} \frac{1}{n} \log \left|a_{n}\right| \leq \min _{r, \rho>0} \log \left(\frac{(1+r)^{14}(r+\rho)^{15}(1+r+\rho)^{15}}{r^{16} \rho^{12}}\right) .
$$

The minimum $\beta$ of the right-hand side is attained at $r=(3 \sqrt{6969}-127) / 224$ and $\rho=(285-\sqrt{6969}) / 336$; therefore we get

$$
\begin{aligned}
\beta & =\log \left(\frac{5^{15}(\sqrt{6969}+285)^{12}(3 \sqrt{6969}+97)^{14}(3 \sqrt{6969}+127)^{16}(5 \sqrt{6969}+343)^{15}}{2^{269} 3^{15} 7^{14} 13^{28} 17^{12}}\right) \\
& <35.4093 .
\end{aligned}
$$

\section{The denominator of $b_{n}(\mathrm{I})$}

In this section we investigate the prime factors $p$ of $T_{n} \equiv b_{n} D_{16 n} D_{18 n}$ satisfying $5 \sqrt{n}<p<15 n$.

After $k$-fold and $(12 n-k)$-fold partial integrations with respect to $x$ and $y$ respectively, we obtain, from (1.2),

$$
\epsilon_{n}=\left(\begin{array}{c}
12 n \\
k
\end{array}\right)^{-1} \iint_{S} \frac{F_{k}(x) G_{k}(y)}{1-x y} d x d y
$$

with

$$
F_{k}(x)=x^{k-12 n}\left(x^{15 n}(1-x)^{15 n}\right)^{(k)} / k !, \quad G_{k}(y)=\left(y^{14 n-k}(1-y)^{14 n}\right)^{(12 n-k)} /(12 n-k) !
$$


for any integer $k \in[0,12 n]$. Note that $F_{k}(x) \in \mathbb{Z}[x]$ and $G_{k}(y) \in \mathbb{Z}[y]$ with $\operatorname{deg}\left(F_{k}\right)=18 n, \operatorname{ord}_{0}\left(F_{k}\right)=3 n, \operatorname{deg}\left(G_{k}\right)=16 n$ and $\operatorname{ord}_{0}\left(G_{k}\right)=2 n$. Then, from Lemma 1.1, (1.3) and (3.1),

$$
a_{k, n} \zeta(2)+b_{k, n}=\left(\begin{array}{c}
12 n \\
k
\end{array}\right) \epsilon_{n}=\left(\begin{array}{c}
12 n \\
k
\end{array}\right)\left\{a_{n} \zeta(2)+b_{n}\right\}
$$

for some $a_{k, n} \in \mathbb{Z}$ and $b_{k, n} \in \mathbb{Z} / D_{16 n} D_{18 n}$. Thus, putting $T_{k, n} \equiv b_{k, n} D_{16 n} D_{18 n}$, we obtain

$$
T_{k, n}=\left(\begin{array}{c}
12 n \\
k
\end{array}\right) T_{n}
$$

since $\zeta(2)$ is irrational. For any prime $p$ let $v_{p}(n)$ be the exponent of $p$ in the resolution of $n$ into its prime factors. Hence, from (3.2),

$$
v_{p}\left(T_{k, n}\right)=[12 \omega]-[12 \omega-\kappa]+v_{p}\left(T_{n}\right)
$$

where $\omega=\{n / p\}$ and $\kappa=\{k / p\}$ for any prime $p>5 \sqrt{n}$. (Here $\{x\}$ denotes the fractional part of $x$.)

On the other hand, it is easily seen from Lemma 1.1 that if $p$ is a common prime factor of all the coefficients of $F_{k}(x)$, or of $G_{k}(y)$, then $p$ becomes a divisor of $T_{k, n}$. More precisely, we have

$$
v_{p}\left(T_{k, n}\right) \geq \min _{0 \leq j \leq 15 n} v_{p}\left(A_{j, k, n}\right)+\min _{0 \leq l \leq 14 n} v_{p}\left(B_{l, k, n}\right)
$$

where

$$
A_{j, k, n}=\left(\begin{array}{c}
15 n \\
j
\end{array}\right)\left(\begin{array}{c}
15 n+j \\
k
\end{array}\right) \quad \text { and } \quad B_{l, k, n}=\left(\begin{array}{c}
14 n \\
l
\end{array}\right)\left(\begin{array}{c}
14 n-k+l \\
12 n-k
\end{array}\right) .
$$

Since $v_{p}\left(A_{j, k, n}\right)=[15 \omega]-[15 \omega-\theta]+[15 \omega+\theta]-[15 \omega+\theta-\kappa] \equiv I(\omega, \theta, \kappa)$, say, and $v_{p}\left(B_{l, k, n}\right)=[14 \omega]-\left[14 \omega-\theta^{\prime}\right]+\left[14 \omega-\kappa+\theta^{\prime}\right]-[12 \omega-\kappa]-\left[2 \omega+\theta^{\prime}\right] \equiv J\left(\omega, \theta^{\prime}, \kappa\right)$, say, for any $p>5 \sqrt{n}$ where $\theta=\{j / p\}$ and $\theta^{\prime}=\{l / p\}$, we have

$$
v_{p}\left(T_{k, n}\right) \geq \min _{0 \leq \theta<1} I(\omega, \theta, \kappa)+\min _{0 \leq \theta^{\prime}<1} J\left(\omega, \theta^{\prime}, \kappa\right)
$$

for any $k \in[0,12 n]$.

We now distinguish four cases, as follows:

CASE I: We take $k=12 n$ giving $\kappa=\{12 \omega\}$. From (3.3), $v_{p}\left(T_{12 n, n}\right)=v_{p}\left(T_{n}\right)$. Suppose now that $2\{15 \omega\}<\{12 \omega\}$. Then clearly

$$
\begin{aligned}
I(\omega, \theta,\{12 \omega\}) & =[15 \omega]-[15 \omega-\theta]+[15 \omega+\theta]-[12 \omega]-[3 \omega+\theta] \\
& \geq 2[15 \omega]-[12 \omega]-[18 \omega]=\{12 \omega\}+\{18 \omega\}-2\{15 \omega\} \\
& >\{18 \omega\} \geq 0
\end{aligned}
$$

for any $\theta \in[0,1)$; hence we have $v_{p}\left(T_{n}\right) \geq 1$ from (3.4), since $I(\omega, \theta, \kappa) \in \mathbb{Z}$. 
CASE II: We take $k=0$ giving $\kappa=0$. From (3.3), $v_{p}\left(T_{0, n}\right)=v_{p}\left(T_{n}\right)$. Suppose now that $2\{14 \omega\}<\{12 \omega\}$. Then

$$
\begin{aligned}
J\left(\omega, \theta^{\prime}, 0\right) & =[14 \omega]-\left[14 \omega-\theta^{\prime}\right]+\left[14 \omega+\theta^{\prime}\right]-[12 \omega]-\left[2 \omega+\theta^{\prime}\right] \\
& \geq 2[14 \omega]-[12 \omega]-[16 \omega]=\{12 \omega\}+\{16 \omega\}-2\{14 \omega\} \\
& >\{16 \omega\} \geq 0
\end{aligned}
$$

for any $\theta^{\prime} \in[0,1)$; hence $\nu_{p}\left(T_{n}\right) \geq 1$ from (3.4).

CASE III: From (3.3) we have always $v_{p}\left(T_{n}\right) \geq v_{p}\left(T_{k, n}\right)-1$. Suppose that $\{2 \omega\}+$ $\{12 \omega\}<1,\{2 \omega\}+\{14 \omega\}<1$ and $2\{15 \omega\}<\{14 \omega\}$. Since $p<14 n$, one can take $k=k(p) \equiv 14 n-[14 n / p] p$; hence $\kappa=\kappa_{\omega} \equiv\{2 \omega\}+\{12 \omega\}$. Then, for any $\theta, \theta^{\prime} \in[0,1)$,

$$
\begin{aligned}
I\left(\omega, \theta, \kappa_{\omega}\right) & =[15 \omega]-[15 \omega-\theta]+[15 \omega+\theta]-[14 \omega]-[\omega+\theta] \\
& \geq 2[15 \omega]-[14 \omega]-[16 \omega]=\{14 \omega\}+\{16 \omega\}-2\{15 \omega\} \\
& >\{16 \omega\} \geq 0
\end{aligned}
$$

and

$$
\begin{aligned}
J\left(\omega, \theta^{\prime}, \kappa_{\omega}\right) & =[14 \omega]-\left[14 \omega-\theta^{\prime}\right]+1+[2 \omega]-\left[2 \omega+\theta^{\prime}\right] \\
& \geq 1+[2 \omega]+[14 \omega]-[16 \omega]=1+\{16 \omega\}-\{2 \omega\}-\{14 \omega\} \\
& >\{16 \omega\} \geq 0 .
\end{aligned}
$$

Hence $v_{p}\left(T_{k(p), n}\right) \geq 2$ from (3.4) and so $v_{p}\left(T_{n}\right) \geq 1$.

CASE IV: The same argument as in Case III can be applied to the following expression:

$$
\epsilon_{n}=\left(\begin{array}{c}
12 n \\
k
\end{array}\right)^{-1} \iint_{S} \frac{U_{k}(x) V_{k}(y)}{1-x y} d x d y
$$

with

$$
U_{k}(x)=\frac{\left(x^{15 n-k}(1-x)^{15 n}\right)^{(12 n-k)}}{(12 n-k) !}, \quad V_{k}(y)=\frac{y^{k-12 n}\left(y^{14 n}(1-y)^{14 n}\right)^{(k)}}{k !}
$$

for any integer $k \in[0,12 n]$, which comes from $k$-fold and $(12 n-k)$-fold partial integrations with respect to $y$ and $x$ respectively. Then, by taking $k=15 n-$ $[15 n / p] p$, it can be seen that $\nu_{p}\left(T_{n}\right) \geq 1$ for any $\omega$ satisfying $\{3 \omega\}+\{12 \omega\}<1$, $\{3 \omega\}+\{15 \omega\}<1$ and $2\{14 \omega\}<\{15 \omega\}$. We have thus proved the following

LEMMA 3.1. Suppose a prime $p \in(5 \sqrt{n}, 15 n)$ satisfies one of the following four conditions: 
(I) $2\{15 \omega\}<\{12 \omega\}$;

(II) $2\{14 \omega\}<\{12 \omega\}$;

(III) $\{2 \omega\}+\{12 \omega\}<1,\{2 \omega\}+\{14 \omega\}<1$ and $2\{15 \omega\}<\{14 \omega\}$;

(IV) $\{3 \omega\}+\{12 \omega\}<1,\{3 \omega\}+\{15 \omega\}<1$ and $2\{14 \omega\}<\{15 \omega\}$.

Then $p$ must divide $T_{n}$.

\section{The denominator of $b_{n}$ (II)}

In this section we investigate prime factors $p \in(5 \sqrt{n}, 15 n)$ of $T_{n}$ satisfying $p^{2} \mid T_{n}$. Suppose now that $\{2 \omega\}+\{12 \omega\} \geq 1$. In the expression (3.1), one can take $k=$ $k(p) \equiv 14 n-[14 n / p] p ;$ thus $\kappa=\tilde{\kappa}_{\omega} \equiv\{2 \omega\}+\{12 \omega\}-1$. Hence, from (3.3), $v_{p}\left(T_{k(p), n}\right)=v_{p}\left(T_{n}\right)$. Suppose further that $\{2 \omega\}+\{14 \omega\}<1$ and $2\{15 \omega\}<\{14 \omega\}$. Then, for any $\theta, \theta^{\prime} \in[0,1)$,

$$
\begin{aligned}
I\left(\omega, \theta, \tilde{\kappa}_{\omega}\right) & =[15 \omega]-[15 \omega-\theta]+[15 \omega+\theta]-1-[2 \omega]-[12 \omega]-[\omega+\theta] \\
& \geq 2[15 \omega]-[14 \omega]-[16 \omega]=\{14 \omega\}+\{16 \omega\}-2\{15 \omega\} \\
& >\{16 \omega\} \geq 0
\end{aligned}
$$

and

$$
\begin{aligned}
J\left(\omega, \theta^{\prime}, \tilde{\kappa}_{\omega}\right) & =[14 \omega]-\left[14 \omega-\theta^{\prime}\right]+[2 \omega]+1-\left[2 \omega+\theta^{\prime}\right] \\
& \geq 1+[2 \omega]+[14 \omega]-[16 \omega]=1+\{16 \omega\}-\{2 \omega\}-\{14 \omega\} \\
& >\{16 \omega\} \geq 0 .
\end{aligned}
$$

Therefore $v_{p}\left(T_{k(p), n}\right) \geq 2$ from (3.4); hence $v_{p}\left(T_{n}\right) \geq 2$.

A similar argument to that above can be applied to the expression (3.5). By taking $k=15 n-[15 n / p] p$, we can show that $\nu_{p}\left(T_{n}\right) \geq 2$ if $\omega$ satisfies $\{3 \omega\}+\{12 \omega\} \geq 1$, $\{3 \omega\}+\{15 \omega\}<1$ and $2\{14 \omega\}<\{15 \omega\}$.

We have thus proved the following

LEMMA 4.1. Suppose a prime $p \in(5 \sqrt{n}, 15 n)$ satisfies one of the following two conditions:

(V) $\{2 \omega\}+\{12 \omega\} \geq 1,\{2 \omega\}+\{14 \omega\}<1$ and $2\{15 \omega\}<\{14 \omega\}$;

(VI) $\{3 \omega\}+\{12 \omega\} \geq 1,\{3 \omega\}+\{15 \omega\}<1$ and $2\{14 \omega\}<\{15 \omega\}$.

Then $p^{2}$ must divide $T_{n}$.

\section{Proof of Theorem 1.2}

Let $\Omega_{1}$ and $\Omega_{2}$ be the sets of $\omega \in[0,1)$ satisfying one of the conditions stated in Lemma 3.1 (I, II, III or IV) and in Lemma 4.1 (V or VI) respectively. Then it is easily 
verified that the set $\Omega_{1}$ consists of the following intervals:

$$
\begin{aligned}
& {\left[\frac{1}{15}, \frac{1}{12}\right),\left[\frac{2}{15}, \frac{1}{6}\right),\left[\frac{1}{5}, \frac{1}{4}\right),\left[\frac{4}{15}, \frac{5}{18}\right),\left[\frac{2}{7}, \frac{5}{16}\right),\left[\frac{5}{14}, \frac{5}{13}\right),\left[\frac{2}{5}, \frac{5}{12}\right),} \\
& {\left[\frac{3}{7}, \frac{4}{9}\right),\left[\frac{7}{15}, \frac{1}{2}\right),\left[\frac{8}{15}, \frac{9}{16}\right),\left[\frac{4}{7}, \frac{7}{12}\right),\left[\frac{3}{5}, \frac{5}{8}\right),\left[\frac{9}{14}, \frac{11}{16}\right),\left[\frac{5}{7}, \frac{3}{4}\right),} \\
& {\left[\frac{11}{14}, \frac{5}{6}\right),\left[\frac{6}{7}, \frac{8}{9}\right), \quad \text { and }\left[\frac{13}{14}, \frac{17}{18}\right) .}
\end{aligned}
$$

Similarly the set $\Omega_{2}$ consists of the following intervals:

$$
\left[\frac{1}{14}, \frac{1}{13}\right),\left[\frac{1}{7}, \frac{2}{13}\right),\left[\frac{3}{14}, \frac{2}{9}\right),\left[\frac{11}{15}, \frac{3}{4}\right),\left[\frac{4}{5}, \frac{13}{16}\right),\left[\frac{13}{15}, \frac{7}{8}\right) \text {, and }\left[\frac{14}{15}, \frac{15}{16}\right) \text {. }
$$

Note that $\Omega_{2} \subset \Omega_{1}$. Putting

$$
\Delta_{i}=\prod_{\substack{p: \text { prime } \\ 5 \sqrt{n}<p<15 n \\\{n / p\} \in \Omega_{i}}} p
$$

for $i=1,2$, it follows from Lemmas 3.1 and 4.1 that the integer $d_{n} \equiv \Delta_{1} \Delta_{2}$ must divide $T_{n}$; that is,

$$
b_{n} \in \frac{d_{n}}{D_{16 n} D_{18 n}} \mathbb{Z}
$$

Note that $d_{n}$ is a divisor of $D_{16 n} D_{18 n}$. Then, as in [6,7], it follows from the prime number theorem that

$$
\lim _{n \rightarrow \infty} \frac{1}{n} \log d_{n}=\int_{\Omega_{1}} d \psi(x)+\int_{\Omega_{2}} d \psi(x) \equiv \gamma, \quad \text { say, }
$$

where $\psi(x)=\Gamma^{\prime}(x) / \Gamma(x)$ is the digamma function. The above Stieltjes integrals can be expressed as sums of values of some elementary functions, by virtue of Gauss' formula. (See, for example, Erdélyi et al. [5, p. 19].) Numerically one obtains

$$
\gamma>9.22875 \text {. }
$$

Now, putting $K_{n}=D_{16 n} D_{18 n} / d_{n} \in \mathbb{Z}$, we have, from (1.3) and (5.1),

$$
K_{n} a_{n} \zeta(2)-K_{n} \epsilon_{n}=-K_{n} b_{n} \in \mathbb{Z} \text {. }
$$

Then we get

$$
\limsup _{n \rightarrow \infty} \frac{1}{n} \log \left|K_{n} a_{n}\right| \leq 34-\gamma+\beta \equiv \sigma, \quad \text { say, }
$$

and

$$
\lim _{n \rightarrow \infty} \frac{1}{n} \log \left|K_{n} \epsilon_{n}\right|=34-\gamma+\alpha \equiv-\tau, \quad \text { say, }
$$


Therefore it follows from (2.1), (2.2) and (5.2) that $\pi^{2}$ has an irrationality measure

$$
1+\frac{\sigma}{\tau}=1-\frac{34-\gamma+\beta}{34-\gamma+\alpha}<\frac{35.4093+36.0223}{9.22875-34+36.0223}<6.3489 .
$$

This completes the proof.

\section{Addendum}

Using the birational diffeomorphism $\tau:(0,1)^{2} \rightarrow(0,1)^{2}$ defined by

$$
\tau(x, y)=\left(1-x y, \frac{1-x}{1-x y}\right),
$$

it follows that the double integral (1.2) can also be written as

$$
\epsilon_{n}=\iint_{S} \frac{x^{17 n}(1-x)^{14 n} y^{15 n}(1-y)^{14 n}}{(1-x y)^{13 n+1}} d x d y .
$$

The transformation $\tau$, satisfying $\tau=\tau^{-1}$, was essentially used in Rhin and Viola [8], although their transformation generates a cyclic group of order 5 . The above expression $\left(^{*}\right)$ gives us further information about the denominator of $b_{n} \in \mathbb{Q}$ so that we can obtain

THEOREM. There exists a positive integer $q_{0}$ such that

$$
\left|\pi^{2}-\frac{p}{q}\right| \gg q^{-5.687}
$$

for all $p \in \mathbb{Z}$ and any integer $q \geq q_{0}$.

PROOF. After $k$-fold and (13n-k)-fold partial integration with respect to $x$ and $y$ respectively in $\left(^{*}\right)$, it can be seen that

$$
v_{p}\left(T_{n}\right) \geq[13 \omega-\kappa]-[13 \omega]+\min _{0 \leq \theta<1} \tilde{I}(\omega, \theta, \kappa)+\min _{0 \leq \theta^{\prime}<1} \tilde{J}\left(\omega, \theta^{\prime}, \kappa\right)
$$

with

$$
\begin{aligned}
\tilde{I}(\omega, \theta, \kappa) & =[14 \omega]-[14 \omega-\theta]+[17 \omega+\theta]-[17 \omega+\theta-\kappa], \\
\tilde{J}\left(\omega, \theta^{\prime}, \kappa\right) & =[14 \omega]-\left[14 \omega-\theta^{\prime}\right]+\left[15 \omega-\kappa+\theta^{\prime}\right]-[13 \omega-\kappa]-\left[2 \omega+\theta^{\prime}\right]
\end{aligned}
$$

for any integer $k \in[0,13 n]$ and for any prime $p>6 \sqrt{n}$, where $\omega=\{n / p\}$, $\kappa=\{k / p\}$. Using this estimate for $v_{p}\left(T_{n}\right)$, Lemmas 3.1 and 4.1 can now be improved as follows. 
LEMMA 1. Suppose a prime $p \in(6 \sqrt{n}, 15 n)$ satisfies one of the conditions (I), (II), (III), (IV), (VII) or (VIII), the latter two being:

(VII) $\{14 \omega\}+\{17 \omega\}<\{13 \omega\}$,

(VIII) $\{2 \omega\}+\{13 \omega\}<1,\{2 \omega\}+\{14 \omega\}<1$ and $\{14 \omega\}+\{17 \omega\}<\{2 \omega\}+\{13 \omega\}$.

Then $p$ must divide $T_{n}$.

LEMMA 2. Suppose a prime $p \in(6 \sqrt{n}, 15 n)$ satisfies one of the conditions (V), (VI) or

(IX) $\{2 \omega\}+\{14 \omega\}<1$ and $1+\{14 \omega\}+\{17 \omega\}<\{2 \omega\}+\{13 \omega\}$.

Then $p^{2}$ must divide $T_{n}$.

The above lemmas can be easily verified as above by taking $k=13 n$ in Case (VII) and $k=15 n-[15 n / p] p$ in Cases (VIII) and (IX). Thus the sets $\Omega_{1}$ and $\Omega_{2}$ can be replaced by

$$
\begin{aligned}
& \tilde{\Omega}_{1}=\Omega_{1} \cup\left[\frac{1}{17}, \frac{1}{16}\right)^{*} \cup\left[\frac{2}{17}, \frac{1}{8}\right) \cup\left[\frac{3}{17}, \frac{3}{16}\right) \cup\left[\frac{9}{17}, \frac{8}{15}\right) \cup\left[\frac{10}{17}, \frac{3}{5}\right) \cup\left[\frac{13}{17}, \frac{10}{13}\right), \\
& \tilde{\Omega}_{2}=\Omega_{2} \cup\left[\frac{5}{17}, \frac{4}{13}\right) \cup\left[\frac{5}{14}, \frac{3}{8}\right) \cup\left[\frac{3}{7}, \frac{7}{16}\right)
\end{aligned}
$$

respectively. Here the contribution of the interval $\left[\frac{1}{17}, \frac{1}{16}\right)^{*}$ to the corresponding Stieltjes integral must be treated differently as follows:

$$
\int_{\left[\frac{1}{17}, \frac{1}{16}\right)^{*}} d \psi(x)=\sum_{n=1}^{\infty} \frac{1}{(16 n+1)(17 n+1)}=\psi\left(\frac{1}{16}\right)-\psi\left(\frac{1}{17}\right)-1,
$$

since $p<15 n$. Consequently we obtain a better exponent $\tilde{\gamma}>10.5383$. Therefore $\pi^{2}$ has an irrationality measure less than

$$
\frac{35.4093+36.0223}{10.5383-34+36.0223}<5.687
$$

which completes the proof.

\section{References}

[1] F. Beukers, 'A note on the irrationality of $\zeta(2)$ and $\zeta(3)$ ', Bull. London Math. Soc. 11 (1979), $268-272$.

[2] D. V. Chudnovsky and G. V. Chudnovsky, Padé and rational approximations to systems of functions and their arithmetic applications Lecture Notes in Mathematics 1052 (Springer, Berlin, 1984) pp. $37-84$. 
[3] _ - 'Transcendental methods and theta-functions', in: Proc. Sympos. Pure Math. 49 (Amer. Math. Soc., Providence, 1989), pp. 167-232.

[4] R. Dvornicich and C. Viola, 'Some remarks on Beukers' integrals', Colloq. Math. Soc. János Bolyai 51 (1987), 637-657.

[5] A. Erdélyi et al., Higher transcendental functions, volume 1 (McGraw-Hill, New York, 1953).

[6] M. Hata, 'Legendre type polynomials and irrationality measures', J. Reine Angew. Math. 407 (1990), $99-125$.

[7] — 'Rational approximations to $\pi$ and some other numbers', Acta Arithmetica 63 (1993), $335-349$.

[8] G. Rhin and C. Viola, 'On the irrationality measure of $\zeta(2)$ ', Ann. Inst. Fourier (Grenoble) 43 (1993), 85-109.

[9] E. A. Rukhadze, 'A lower bound for the approximation of $\ln 2$ by rational numbers', Vestnik Moskov. Univ. Ser. I Mat. Mekh. 6 (1987), 25-29 in Russian.

Institute of Mathematics

Yoshida College

Kyoto University 